\title{
An alternative image quality assessment method for blurred images
}

\author{
H. Boztoprak
}

\begin{abstract}
This study proposes a no-reference image quality assessment method for blurred images. In this approach, first, a discrete wavelet transform was applied to the sample images and then the results were decomposed into four subbands. This was followed by the calculation of the spatial frequencies of high-high $\left(\mathrm{HH}_{2}\right)$ and low-low $\left(\mathrm{LL}_{2}\right)$ subbands. Then the ratio of spatial frequencies of $\mathrm{HH}_{2}$ and $\mathrm{LL}_{2}$ subbands was calculated. Information about the image quality was obtained by using this ratio, with lower values indicative of better image quality. The study aims to investigate whether the proposed method is capable of measuring the image quality. The proposed technique was tested on the standard images. Three different images were used, of which each one was distorted with the same type and amount of noise. Motion noise, blurring and sharpening was applied to distort the images. The performance of the proposed method was evaluated and compared with eight representative image quality measures. This provides a meaningful comparison across different types of image distortions. Then, the cameraman image was also blurred with two different noises: Gaussian and disk-shaped blur. The varying amount of blur was compared with Universal Image Quality Index (UIQI) values of the cameraman image. The method gives good results in different resolutions as well. Its computation is easy, independent of viewing conditions.
\end{abstract}

Index Terms - Blurring, Image Quality, Spatial Frequency, Wavelet Transform

\section{INTRODUCTION}

$\mathrm{I}$ MAGE quality is a characteristic of an image that measures the detected image corruption. The quality assessment is an important part of image processing and vision algorithms. For measure amount of degradation in compressed image, filtering and quality enhancement systems are often used. Image compression reduces the quality of the image and some measure to quantify this image degradation is required.

Image quality assessment can be done subjectively or objectively. Objective image visual quality analysis can be into no-reference $(\mathrm{NR})$, reduced-reference (RF) and fullreference (FR) based on the availability of the reference image. In FR metrics, the quality of a test image is measured by comparing it with a reference image. RR methods have partial information from the reference image. However, NR methods try to estimate the quality of an image without any reference. The aim of objective quality assessment research is to provide quality metrics that can predict video and image quality automatically [1].

H. Boztoprak, is with Department Computer Program, Alaaddin Keykubat University, Akseki Vocational School, Antalya, Turkey, (e-mail: halimeboz@yahoo.com).
Subjective methods are impractical for real time implementation, because there are costly and time consuming [2]. In human vision system (HSV) based metrics, difference between the test and the reference images is standardized according to its visibility, as determined by psychophysics of human perception.

There are many objective image quality measure methods in the literature. The MSE (Mean Squared Error) and the PSNR (Peak Signal-to-Noise Ratio) are the two most common objective assessment methods [3]. The MSE, SNR and PSNR are computed quickly. However, these assessment methods are not always the best choice, especially if a comparison will be performed against the human perception of the image quality [4]. A mathematically defined universal image quality index is proposed [5]. This approach does not depend on the images being tested, the individual observers or the viewing conditions. It must be convenient to different image processing applications and supply significant comparison across various types of image distortions. UIQI and SSIM are more correct and consistent than PSNR and MSE despite they cost more [6]. De-noising performances are quantitatively measured by the PSNR, MSE, RMSE, IEF (Measure of Enhancement Factor) [7-9].

Blurring is one of the most common distortions encountered in image processing applications. It can be caused by lot of reasons like camera shake, defocus, motion etc. Extreme blur in an image creates problem for user to classify and identify objects in an image. Numerous design strategies have been utilized by different researchers in developing image quality assessment techniques for blurred images [10]. Calculation of edge-width [11], histogram based assessment [12, 13], power spectrum based assessment [14], wavelet based techniques $[15,16]$, and neural network-based methods [4] are among these approaches. A no reference blur image quality metric was introduced in [17]. This approach was based on wavelet transform. It was examined edges through a multiresolution decomposition. In [18] study, the input image is decomposed via a three-level separable discrete wavelet transforms (DWT). The log-energies of the DWT subbands are estimated. A scalar index is calculated using a weighted average of these log-energies.

This study proposes a no-reference image quality assessment method for blurred images. The approach is independent from the images being tested, the individual observers or the viewing conditions. We present a simple and effective method. Its computation is easy, independent of viewing conditions. This supplies a meaning comparison across various types of image distortions. The achievement of the proposed method was estimated and compared with the other representative quality assessment. 


\section{MATERIAL AND METHODS}

\subsection{Image Quality Measures}

In the objective approach, the image quality is assessed by using a mathematical formula that attempts to quantify the amount of image distortion.

\subsubsection{The signal to noise ratio (SNR) and Peak Signal to Noise Ratio}

SNR and PSNR are a mathematical measure of image quality based on the pixel differences between two images $[19,20]$. The SNR measure is a calculation of the quality of a reconstructed image compared with the original image. The whole idea is to obtain a single number that reflects the quality of the image by computing the ratio of the signal power to the noise power. The PSNR is the rate between the maximum possible power of a signal and the power of corrupting noise. The PSNR is expressed in logarithmic level. The mathematical term for it is:

$$
\mathrm{PSNR}=20 \log _{10}\left(\frac{\mathrm{MAX}_{\mathrm{f}}}{\sqrt{\mathrm{MSE}}}\right)
$$

MAX $_{\mathrm{f}}$ : The maximum signal value that exists in the original image

\subsubsection{Mean Squared Error (MSE)}

MSE is a measure of control and quality. The MSE is defined as follows

$$
\mathrm{MSE}=\frac{1}{\mathrm{M}^{*} \mathrm{~N}} \sum_{a} \sum_{b}[A(a, b)-W(a, b)]^{2}
$$

where $\mathrm{A}(\mathrm{a}, \mathrm{b})$ is the original image and $\mathrm{W}(\mathrm{a}, \mathrm{b})$ is the distorted image that contain $\mathrm{M} \times \mathrm{N}$ pixels.

\subsubsection{Universal Image Quality Index (UIQI)}

The UIQI is a mathematically defined measurement, that is easy to compute and independent of viewing conditions. In 2002, Wang and Bovik proposed this measure [5], it breaks the comparison between distorted and original image into three comparisons: contrast, structural, luminance and comparisons as in (3), (4), and (5). The dynamic range for the quality index is $[-1 ; 1]$, where 1 represents a perfect image quality.

$$
\begin{aligned}
& \mathrm{l}(\mathrm{x}, \mathrm{y})=\frac{2 \mu_{x} \mu_{y}}{\mu_{x}^{2}+\mu_{y}^{2}} \\
& \mathrm{c}(\mathrm{x}, \mathrm{y})=\frac{2 \sigma_{x} \sigma_{y}}{\sigma_{x}^{2}+\sigma_{y}^{2}} \\
& \mathrm{~s}(\mathrm{x}, \mathrm{y})=\frac{2 \sigma_{x y}}{\sigma_{x}+\sigma_{y}}
\end{aligned}
$$

where $\mu_{x} \mu_{y}$ represents the mean values of distorted and original images. And $\sigma_{y} \sigma_{x}$ represents the standard deviation of distorted and original images, and $\sigma_{x y}$ is the covariance of both images. The UIQI is given in (6).

$$
\begin{gathered}
\mathrm{UIQI}(\mathrm{x}, \mathrm{y})=\mathrm{I}(\mathrm{x}, \mathrm{y}) \cdot \mathrm{c}(\mathrm{x}, \mathrm{y}) \cdot \mathrm{s}(\mathrm{x}, \mathrm{y}) \\
=\frac{4 \mu_{x} \mu_{y} \mu_{x y}}{\left(\mu_{x}^{2}+\mu_{y}^{2}\right)\left(\sigma_{x}^{2}+\sigma_{y}^{2}\right)}
\end{gathered}
$$

\subsubsection{Root Mean Squared Error (RMSE)}

MSE is a tool to quantify the difference between a copy and the true value of the amount being calculated. In statistic it is defined as a risk function [4]. Mathematically it is expressed as:

$$
\text { RMSE }=\sqrt{\frac{1}{X Y} \sum_{x=0}^{X} \sum_{y=1}^{Y}\left[I(x, y)-I_{c}(x, y)\right]^{2}}
$$

where $I(x, y)$ is the original image and $I_{c}(x, y)$ is the distorted image. $\mathrm{X}$ and $\mathrm{Y}$ are the sizes of the image.

\subsubsection{Structural Similarity (SSIM)}

The distorted and original images are divided into blocks of size $8 \times 8$ and then the blocks are converted into vectors. Then, two standard derivations, two means and one covariance value are computed as in (8), (9), and (10) [5].

$$
\begin{gathered}
\mu_{x}=\frac{1}{T} \sum_{i=1}^{T} x_{i} \mu_{y}=\frac{1}{T} \sum_{i=1}^{T} y_{i} \\
\sigma_{x}^{2}=\frac{1}{T-1} \sum_{i=1}^{T}\left(x_{i}-\bar{x}\right)^{2} \sigma_{y}^{2} \\
=\frac{1}{T-1} \sum_{i=1}^{T}\left(y_{i}-\bar{y}\right)^{2} \\
\sigma_{x y}^{2}=\frac{1}{T-1} \sum_{i=1}^{T}\left(x_{i}-\bar{x}\right)\left(y_{i}-\bar{y}\right)
\end{gathered}
$$

Structural similarity index measure between images $\mathrm{x}$ and $\mathrm{y}$ is given by (11).

$$
\operatorname{SSIM}(\mathrm{x}, \mathrm{y})=\frac{\left(2 \mu_{x} \mu_{y}+c_{1}\right)\left(2 \sigma_{x y}+c_{2}\right)}{\left(\mu_{x}^{2}+\mu_{y}^{2}+c_{1}\right)\left(\sigma_{x}^{2}+\sigma_{y}^{2}+c_{2}\right)}
$$

where $c_{1}$ and $c_{2}$ are constants.

Like in UIQI, SSIM is applied locally using sliding window of size $\mathrm{B} \times \mathrm{B}$ that moves pixel by pixel horizontally and vertically covering all the rows and columns of the image, starting from top left corner of the image [6].

2.1.6. The measure of enhancement (EME) and measure of enhancement factor (EMF)

EME and EMF are defined in equation (4) and (5) respectively. EME (measure of enhancement) has been developed by Agaian et. al. [21]. 


$$
\operatorname{EME}(\mathrm{e})=\frac{1}{k_{1} k_{2}} \sum_{m=1}^{k_{1}} \sum_{l=1}^{k_{2}} 20 \ln \left(\frac{I_{\text {max }}^{l, m}}{I_{\text {min }}^{l, m}}\right)
$$

where, the image is divided into $\mathrm{k}_{1} \mathrm{k}_{2}$ blocks, $\alpha$ is a constant, $I_{\max }^{l, m}$ and $I_{\min }^{l, m}$ are the maximum and minimum values of the pixels in each block of the enhanced image. EMF between input image and output image is described as:

$$
\mathrm{EMF}=\frac{\mathrm{EME}_{\text {output }}}{\mathrm{EME}_{\text {input }}}
$$

\subsubsection{Mean absolute error (MAE)}

MAE is described as below [22];

$$
\operatorname{MAE}(r, e)=\frac{1}{M N} \sum_{i=0}^{M-1} \sum_{j=0}^{N-1}\lfloor n(i, j)\rfloor
$$

\subsection{Proposed Method}

In this study, first, a discrete wavelet transform (DWT) was applied to the sample images. Multi-resolution filter banks and particular wavelet filters are used in this transform for the analysis and reconstruction of signals. The different output signals of the decomposition filter bank are called as sub-bands. A filter bank consists of filters that separate a signal from the frequency bands [23]. Two-level 2D transform operations were computed by using filter banks. The high-high $\left(\mathrm{HH}_{1}\right)$, high-low $\left(\mathrm{HL}_{1}\right)$, low-high $\left(\mathrm{LH}_{1}\right)$, and low-low $\left(\mathrm{LL}_{1}\right)$ subbands are one level decomposition of $2 \mathrm{D}$ DWT. After finishing the first level decomposition, second level decomposition was performed [24].

And, as a result of this transform, it was decomposed into four sub-bands: $\mathrm{HH}_{2}, \mathrm{HL}_{2}, \mathrm{LH}_{2}$ and $\mathrm{LL}_{2}$. This was followed by the calculation of the spatial frequencies of $\mathrm{HH}_{2}$ and $\mathrm{LL}_{2}$ sub-bands using equation (5)-(7). For an $\mathrm{M} \times \mathrm{N}$ sized image, the spatial frequency is calculated as follows: where, RF is the row frequency, and CF is the column frequency. Spatial frequency indicates the overall information level in an image and measures the variation of pixels [25]. It is usually used with wavelet transform in image fusion studies [26, 27]. In [28], wavelet transform and spatial frequency was used to calculate iterative value of cellular neural network. The spatial frequency needs to be decreased if the images are blurred more. And, a higher value of spatial frequency indicates higher quality and contrast of the image.

$$
\begin{aligned}
& S F=\sqrt{(C F)^{2}+(R F)^{2}} \\
& C F=\sqrt{\frac{1}{M N} \sum_{n=1}^{N} \sum_{m=2}^{M}[F(m ; n)-F(m-1, n)]^{2}} \\
& R F=\sqrt{\frac{1}{M N} \sum_{m=1}^{M} \sum_{n=2}^{N}[F(m ; n)-F(m, n-1)]^{2}}
\end{aligned}
$$

Then the ratio of spatial frequencies of $\mathrm{HH}_{2}$ and $\mathrm{LL}_{2}$ subbands was calculated. Information about the image quality was obtained by using this ratio. And, the value was compared with known image sharpness/blur measures.

\section{EXPERIMENTAL RESULTS}

The test images used for our analysis shown were in Fig. 1 (a) Cameraman, (b) Lena and (c) Peppers. Three different images were used, of which each one was distorted with the same type and amount of noise. Motion noise, blurring and sharpening was applied to distort the images. Fig. 2 shows (a) original cameraman image, (b) motion noise, (c) blurring and (d) sharpening image.

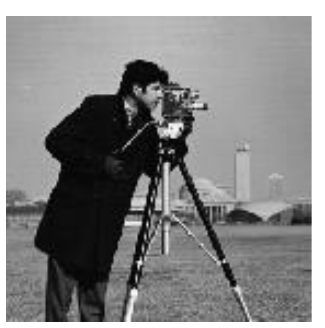

(a)

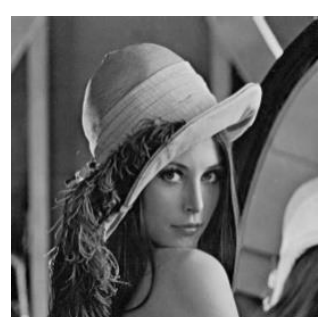

(b)

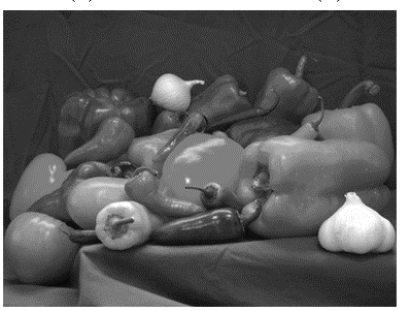

(c)

Fig. 1. Examples of test images used for our analysis (a) Cameraman, (b) Lena and (c) Peppers

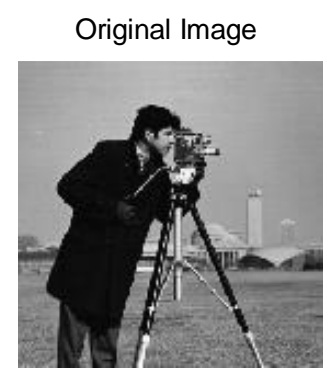

(a)

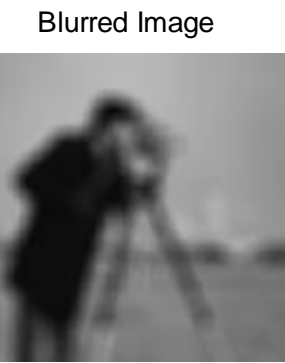

(c)

\section{Motion Blurred Image}

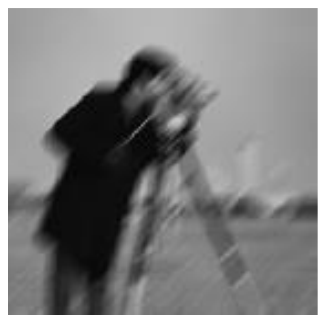

(b)

Sharpened Image

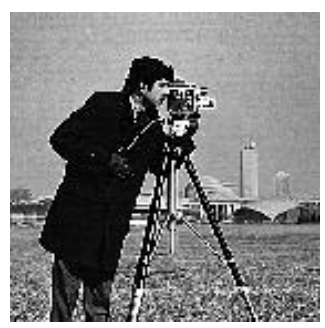

(d)
Fig. 2. (a) Original cameraman image, 256x256, 8 bits/pixel, (b) motion, (c) blurring and (d) sharpened image 
First image used was the 8 bit $256 \times 256$ grayscale cameraman image. After applying some distortion to the original images, the image quality assessments were performed on these distorted images and the results were compared.

The image quality assessment for the cameraman image is shown in the results in Table 1 . Table 1 shows the UIQI, PSNR, MSE, RMSE, EME, EMF, MAE, SNR and SIMM values, calculated between original and distorted images, respectively. Proposed methodology utilizes a no-reference image approach.

TABLE 1

COMPARISON OF CAMERAMAN IMAGE QUALITY MEASURES

\begin{tabular}{|l|c|c|c|}
\hline \multicolumn{1}{|c|}{ Cameraman } & motion & blurred & sharpened \\
\hline Proposed & 3.5203 & 14.1384 & 1.1646 \\
\hline UIQI & 0.2100 & 0.1097 & 0.5678 \\
\hline PSNR & 19.8642 & 18.9878 & 19.5906 \\
\hline MSE & 676.1797 & 827.3670 & 720.1431 \\
\hline RMSE & 26.0035 & 28.7640 & 26.8355 \\
\hline EMF & 0.42 & 0.28 & 0.58 \\
\hline MAE & 14.0620 & 16.344 & 15.4276 \\
\hline SNR & -4.5376 & -5.413 & -4.8111 \\
\hline SIMM & 0.6069 & 0.5508 & 0.6987 \\
\hline
\end{tabular}

The proposed algorithm was also tested on the Lena image. Lena image was an 8 bit 256x256 grayscale image. Assessment values are shown in Table 2.

TABLE 2 .

COMPARISON OF LENA IMAGE QUALITY MEASURES

\begin{tabular}{|l|c|c|c|}
\hline Lena(256x256) & motion & blurred & sharpened \\
\hline Proposed & 3.8160 & 15.3463 & 1.2064 \\
\hline UIQI & 0.4214 & 0.2290 & 0.6718 \\
\hline PSNR & 21.1862 & 19.6975 & 20.7449 \\
\hline MSE & 498.7276 & 702.6348 & 552.0695 \\
\hline RMSE & 22.3322 & 26.5073 & 23.4962 \\
\hline EMF & 0.44 & 0.28 & 0.53 \\
\hline MAE & 14.1988 & 18.1324 & 14.2617 \\
\hline SNR & -2.9832 & -4.4718 & -3.4245 \\
\hline SIMM & 0.6034 & 0.4889 & 0.7026 \\
\hline
\end{tabular}

Some processing was applied to the Peppers Image. The Peppers image was 8 bit and 1200x1200 grayscale image. Assessment values are shown in Table 3.

TABLE 3.

COMPARISON OF PEPPERS IMAGE QUALITY MEASURES

\begin{tabular}{|l|c|c|c|}
\hline $\begin{array}{c}\text { Peppers } \\
(\mathbf{1 2 0 0 x 1 2 0 0 )}\end{array}$ & motion & blurred & Sharpened \\
\hline Proposed & 10.3359 & 17.2360 & 3.0954 \\
\hline UIQI & 0.4176 & 0.3801 & 0.6593 \\
\hline PSNR & 33.6666 & 34.0125 & 41.5911 \\
\hline MSE & 28.1720 & 26.0151 & 4.5433 \\
\hline RMSE & 5.3077 & 5.1005 & 2.1315 \\
\hline EMF & 0.44 & 0.32 & 0.62 \\
\hline MAE & 1.8506 & 1.8128 & 1.2749 \\
\hline SNR & 5.3962 & 5.7421 & 13.3207 \\
\hline SIMM & 0.9679 & 0.9712 & 0.9988 \\
\hline
\end{tabular}

For good metric, the values of PSNR, SNR, UQI, EMF and SSIM should be high. Also, the values of MSE, RMSE, and MAE should be low. RMS is obtained by the square root of the MSE. The small is preferred. The MSE, SNR and PSNR are fast to compute, and hence widely used. However, this measures are not always the best choice, especially if a comparison against human perception of the image quality is done. Although there is a clear visual difference between these images, the difference is not very good reflected in the quality score given by the PSNR and the MSE. The SSIM and UIQI are visually more consistent with the image quality scores, according to the results presented in the Tables. The dynamic range for UIQI is $[-1 ; 1]$, where 1 represents a perfect image quality.

The clarity of image is expected to decrease when a motion or blur distortion is applied. And, the clarity of image is expected to increase when a sharpening is applied. Accordingly, the SIMM and UIQI parameters provide the scores in each of the Tables. The proposed method may give information about the clarity, inversely proportional to these two parameters. Proposed value decreases with increasing clarity. A lower proposed value indicates a better quality image. So, it can give information about the image. The higher this value is so much noise.

The cameraman image was also blurred with two different noises: Gaussian and disk-shaped blur. First, the cameraman image was blurred with Gaussian blur at different values of sigma $=0.5,1.0,2.0,2.5$. Then, the image was further blurred with disk-blur with se $=5,10,12,15$. The blurred images and respective proposed values changed depending on the noise levels are shown in Fig.3 (a). Fig.3 (a) indicates the relationship between the proposed image quality assessment and the amount of blurring applied to the cameraman image for different values of sigma.

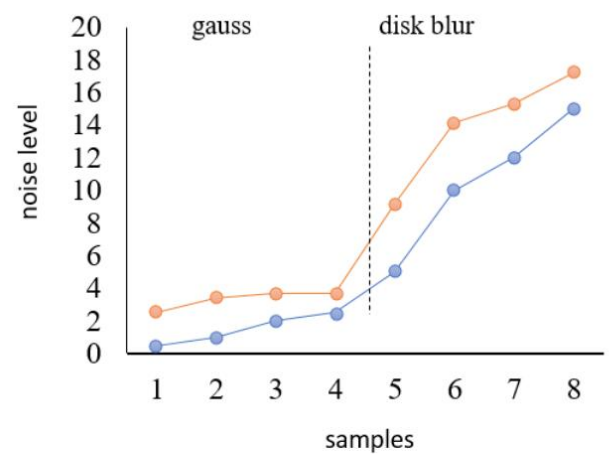

\section{- image quality assessment with proposed method images blurred at different values}

(a)

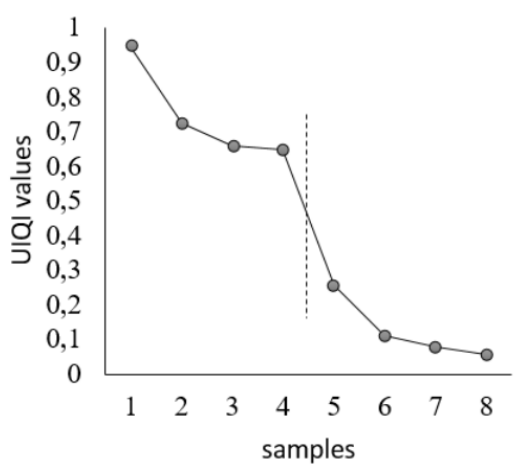

(b)

Fig. 3. (a) Shown that changed proposed value with noise, b) shown that changed UIQI value with noise. 
The value of proposed value increases as the quality of image decreases in all cases. This trend is also seen in the images blurred with different disk-shape blur values. The proposed values increase with increasing noise. Fig. 3 (b) shows UIQI values of the cameraman image with varying amount of blur. An important observation is that the proposed value increases with the amount of blur in an image.

\section{CONCLUSIONS}

It is hard to get the same quality score even if the same level of distortion is applied to different images. Most of the image quality metrics are based on the difference between the distorted and the original image. In this study, we have discussed an objective quality assessment approach that requires no reference image. This method was tested on three different grayscale images. Test images were generated from the original images by distorting with noise. The proposed values obtained without reference images were compared with other image quality assessment parameters. It has a low computational complexity, that is, it is fast and easy to implement. This assessment method gives good results in variable quality of input images as well. And also, it is capable of giving information about the blurred images. The method has been shown to provide information about the quality of the image in a simple way without reference image. The method enables us to measure the noise level. Further studies, using this information about the quality of the image, image enhancement / repair operations can be done.

\section{REFERENCES}

[1] Z. Wang, A. C. Bovik, \& L. Lu, "Why is image quality assessment so difficult?" Acoustics, Speech, and Signal Processing (ICASSP), 2002 IEEE International Conference on. Vol. 4. IEEE, 2002.

[2] A. Zaric, et al. "Image quality assessment-comparison of objective assessment with results of subjective test", pp 113-118, Sep. 2010.

[3] M. Jiang, Digital Image Processing. Department of Information Science, School of Mathematics, Peking University, 2003.

[4] A. Havstad, Image quality assessment using artificial neural networks. MSc, Engineering and Mathematics, Edith Cowan University, 2004.

[5] Z. Wang \& A.C. Bovik, "A universal image quality index." Signal Processing Letters, IEEE 9.3 (2002): 81-84.

[6] Al-Najjar, Yusra AY. Dr. Der Chen Soong, "Comparison of Image Quality Assessment: PSNR, HVS, SSIM, UIQI"." International Journal of Scientific \& Engineering Research 3.8 (2012): 1.

[7] N. Ramanaiah \& S. Kumar, (2013). Removal of high density salt and pepper noise in images and videos using denoising methods.

[8]R. Arumugham, K. Vellingiri, W. F. Habeebrakuman, \& K. Mohan, (2012). A New Denoising Approach for the Removal of Impulse Noise from Color Images and Video Sequences. Image Analysis \& Stereology, 31(3), 185-191.

[9] Om, H., \& Biswas, M. (2015). A generalized image denoising method using neighbouring wavelet coefficients. Signal, Image and Video Processing, 9(1), 191-200.

[10] De, Kanjar, and V. Masilamani. "Image Sharpness Measure for Blurred Images in Frequency Domain." Procedia Engineering 64 (2013): 149-158.

[11] Ong EePing, et al. "A no-reference quality metric for measuring image blur." Signal Processing and Its Applications, 2003. Proceedings. Seventh International Symposium on. Vol. 1. Ieee, 2003.

[12] N.K. Chern, N.P.A. Neow, M.H. Ang Jr, "Blur determination in the compressed domain using DCT information", In Proc. IEEE Int. Conf. Robotics and Automation, 3, pp. 2791-96, 2001.

[13] Y. Yildiray. "A histogram based image quality index." Przegl A Elektrotechniczny Electr. Rev. NR 7a 88 (2012): 126-129.

[14] Nill, Norman B., and Brian Bouzas. "Objective image quality measure derived from digital image power spectra." Optical engineering 31.4 (1992): 813-825.
[15] Ferzli, Rony, and Lina J. Karam. "No-reference objective wavelet based noise immune image sharpness metric." Image Processing, 2005. ICIP 2005. IEEE International Conference on. Vol. 1. IEEE 2005.

[16] Dumic, Emil, Sonja Grgic, and Mislav Grgic. "New image-quality measure based on wavelets." Journal of Electronic Imaging 19.1 (2010): 011018-011018.

[17] Kerouh, F., A. Serir. "A No Reference Quality Metric for Measuring Image Blur In Wavelet Domain." International journal of Digital Information and Wireless Communication 1.4 (2012): 767-776.

[18] Vu, Phong V., and Damon M. Chandler. "A fast wavelet-based algorithm for global and local image sharpness estimation." Signal Processing Letters, IEEE19.7 (2012): 423-426.

[19] Avcybab, Y., Image quality statistics and their use in stange analysis and compression, Ph.D, Bogazici University, İstanbul, Turkey, 2001.

[20] Rafael, C., Gonzalez Woods and Richard, E., Digital Image Processing, Addison-Wesley Publishing Company, 1992.

[21] Lal, Shyam, and Rahul Kumar. "Enhancement of Hyperspectral Rea World Images Using Hybrid Domain Approach." International Journal of Image, Graphics and Signal Processing (IJIGSP) 5.5 (2013): 29.

[22] Planitz, B., and A. Maeder. "Medical image watermarking: A study on image degradation." Proc. Australian Pattern Recognition Society Workshop on Digital Image Computing, WDIC. 2005.

[23] R.J.E. Merry, Steinbuch M., van de Molengraft M.J.G., Wavelet theory and applications literature study, Eindhoven University of Technology, Department of Mechanical Engineering, Control Systems Technology Group, 2005.

[24] Dia, Dhaha, et al. "Multi-level discrete wavelet transform architecture design." Proceedings of the world congress on engineering. Vol. 1. 2009.

[25] A. Eskicioglu, M., and Paul S. Fisher. "Image quality measures and their performance." Communications, IEEE Transactions on 43.12 (1995): 2959-2965.

[26] Maddali R., Prasad K.S., Bindu C.H., Discrete wavelet transform based medical image fusion using spatial frequency technique, Int $\mathrm{J}$ of Sys Alg \& App (IJSAA) 2012; 2: 2277-2677.

[27] Jain, Atika, P. M. Kanjalkar, and J. V. Kulkarni. "Estimation of image focus measure and restoration by Wavelet." Intelligent Networks and Intelligent Systems (ICINIS), 2011 4th International Conference on. IEEE, 2011.

[28] H. Boztoprak, Y. Özbay, A new method for segmentation of microscopic images on activated sludge. Turk J Elec Eng \& Comp Sci, 23.Sup. 1 (2015): 2253-2266.

\section{BIOGRAPHY}

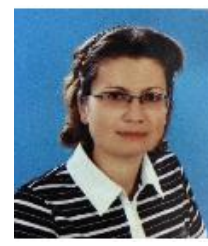

Halime BOZTOPRAK was born in Konya, Turkey in 1982. She received B.Sc. degree in 2004 and the M.Sc. degree in 2007 from the University of Süleyman Demirel, Isparta, and the Ph.D. degree in 2014 from the University of Selçuk, Konya, Turkey. Her current research interests are digital image processing, artificial neural networks, cellular neural network. 